\title{
Association of Relative Leucocyte Telomere Length and Gene Single Nucleotide Polymorphisms (TERT, TRF1, TNKS2) in Laryngeal Squamous Cell Carcinoma
}

\author{
PAULIUS VAICIULIS ${ }^{1}$, RASA LIUTKEVICIENE ${ }^{2}$, VYKINTAS LIUTKEVICIUS ${ }^{1}$, \\ ALVITA VILKEVICIUTE ${ }^{2}$, GRETA GEDVILAITE ${ }^{2}$ and VIRGILIJUS ULOZA ${ }^{1}$ \\ ${ }^{1}$ Department of Otorhinolaryngology, Lithuanian University of Health Sciences, Kaunas, Lithuania, \\ ${ }^{2}$ Neuroscience Institute, Lithuanian University of Health Sciences, Kaunas, Lithuania
}

\begin{abstract}
Background/Aim: The study aimed to evaluate associations of relative leukocyte telomere length (LTL) and polymorphisms of telomere length-associated genes TERT (rs2736098), TERT-CLPTM1L (rs401681), TRF1 (rs1545827, rs10107605) and TNKS2 (rs10509637, rs10509639) in patients with laryngeal squamous cell carcinoma (LSCC). Materials and Methods: The study consisted of 300 patients with LSCC and 369 healthy control subjects. Genotyping and relative LTL measuring were carried out using qPCR. Results: Relative LTL was statistically significantly shorter in the G3 (tumor differentiation grade) subgroup of patients with LSCC compared to the $G 1$ and G2 subgroups. Significant differences were found in genotype distributions of TERT rs401681 and TNKS2 rs10509639 between the study groups. TERT rs401681 C/T and T/T genotypes were associated with approximately $30 \%$ decreased odds of LSCC development. Conclusion: LTL was shorter in the G3 subgroup compared to the G2 and G1 subgroups of LSCC patients. TERT rs401681 and its $C / T$ and T/T genotypes were associated with decreased odds of overall LSCC development.
\end{abstract}

Laryngeal squamous cell carcinoma (LSCC) represents one of the most common upper respiratory tract tumors and ranks 20th among all oncological diseases in Europe (1). According to published data, LSCC accounts for $2.4 \%$ of all

This article is freely accessible online.

Correspondence to: Paulius Vaiciulis, Department of Otorhinolaryngology, Lithuanian University of Health Sciences, Eiveniu 2, Kaunas, Lithuania. Tel: +37 065297454, e-mail: paulius.vaiciulis@1smuni.lt

Key Words: Leucocyte telomeres, TERT, TRF1, TNKS2, laryngeal carcinoma, gene polymorphisms. newly diagnosed malignancies, of which more than $95 \%$ are diagnosed with squamous cell carcinoma (2). The incidence rate of LSCC has decreased almost twofold from 1995 to 2015 (from 4.6/100,000 inhabitants to $2.4 / 100,000$ inhabitants, respectively), but the mortality rate due to this pathology has not changed during the same period (3). The etiology and pathogenesis of LSCC are complex and still poorly recognized. LSCC represents a heterogeneous disease whose pathogenesis is a multistep and multifactorial process that involves both environmental and genetic factors. Therefore, a better understanding of the LSCC pathogenesis requires a comprehensive research of biological and genetic markers of this disease.

In recent years, attention has been increasingly focused on investigations of telomeres and telomere length regulating genes, due to their importance in aging and development of chronic diseases and malignant tumors (4-7). The name telomere itself originates from the Greek word "telos", which means "end", and "meros", which means "part" (8). In eukaryotic cells, telomeres are located at the ends of chromosomes in association with a protein complex (9). Telomeres are specialized DNA structures composed of several thousand DNA repeats of TTAGGG nucleotides (10). These DNA structures are essential for chromosome integrity and genomic stability through prohibiting nucleolytic degradation, chromosomal end-to-end fusion and irregular recombination $(9,11)$. Telomere dysfunction takes an important part in the carcinogenesis process. The shortening of telomeres by initiating apoptosis of senescent cells (telomere protection) is thought to be a mechanism of cancer suppression and constitutes a barrier to uncontrolled proliferation of cells (12). Loss of telomere protection can lead to telomere crisis, which can lead to genomic instability and induce carcinogenesis (12). Several studies have shown an association between changes in peripheral venous blood leucocyte telomere length (LTL) and cancer development (13). Raquel et al. have found the association between short 
telomeres and head and neck cancer development (14). However, the literature data on this matter are rather controversial. Particularly, there is a lack of data on the role of telomere length in LSCC development.

Several factors are known to determine the function of telomeres, which may also influence carcinogenesis. The telomerase complex and the shelterin complex, as well as tankyrase, are important for telomere function. Extensive studies of telomerase activity revealed that it could be a predictor of cancer risk (15). Telomerase complex counteracts telomere shortening and plays an important role in cells becoming immortal. These structures consist of both a catalytic protein subunit [telomerase reverse transcriptase (TERT)] and a human telomerase RNA component that acts as a template for inserting a sequence of the TTAGGG repeats at the 3 'end of telomeric DNA (16). Singlenucleotide polymorphisms (SNP) of TERT and TERT CLPTMIL demonstrated the relationship with cancer risk, including lung, urinary bladder, prostate, cervix and hepatocellular malignancies (17-21). However, only one paper revealing the association between polymorphisms of the TERT-CLPTMIL and pharynx-larynx cancer has been published so far (22).

Telomere binding complexes, such as shelterin, are known to protect telomeres from DNA repair mechanisms, as well as to regulate telomerase activity $(23,24)$. Six protein subunits (TRF1, TRF2, RAP1, TIN2, TPP1, and POT1) that form the shelterin complex are supposed to be involved in this activity (24). TRF1 in co-action with both Tankyrase 1 (TNKS1/ARTD5/PARP5a) and Tankyrase 2 (TNKS2/ARTD6/ $P A R P 5 b$ ) controls telomere length negatively (telomere shortening is a result of TRF1 overexpression, while telomere lengthening is due to dominant-negative TRF1) $(25,26)$. Only a few studies have investigated associations between SNPs of TRF1 and TNKS2 and different carcinomas $(27,28)$. Wang et $a l$. have found that TNKS2 rs 1340420 SNP was associated with lower non-small cell lung cancer risk, whereas TNKS2 rs1770474 SNP was associated with higher squamous-cell carcinoma risk, suggesting that these two SNPs may be useful as predictors of risk of developing these malignancies (28). Varadi et al. have investigated the relationship between polymorphisms in telomere-associated genes (including TRF1 and TNKS2) and breast cancer. Based on this study, authors concluded that none of the SNPs represents a valuable prognostic marker for breast cancer (27). However, a definitive conclusion on this matter is yet to be reached. To the best of our knowledge, there is no data in the literature regarding the associations between the aforementioned SNPs, LTL and LSCC.

The aim of the present study was to evaluate associations of relative LTL and SNPs of telomere length-associated genes TERT (rs2736098), TERT-CLPTMIL (rs401681), TRF1 (rs1545827, rs10107605) and TNKS2 (rs10509637, rs 10509639) in patients with LSCC.
Table I. Demographic characteristics of study subjects.

\begin{tabular}{|c|c|c|c|}
\hline \multirow[t]{2}{*}{ Characteristic } & \multicolumn{2}{|c|}{ Group } & \multirow[t]{2}{*}{$p$-Value } \\
\hline & $\begin{array}{l}\text { LSCC } \\
n=300\end{array}$ & $\begin{array}{c}\text { Control group } \\
n=369\end{array}$ & \\
\hline Male, n (\%) & $298(96.3)$ & 357 (96.7) & $0.770 *$ \\
\hline Female n $(\%)$ & $11(3.7)$ & $12(3.3)$ & \\
\hline Age years; mean (SD) & $62.8(8.8)$ & $62.5(14.3)$ & $0.746 * *$ \\
\hline Stage, n (\%) & & - & - \\
\hline I & $90(30.8)$ & & \\
\hline II & $66(22.6)$ & & \\
\hline III & $53(18.2)$ & & \\
\hline IV & $83(28.4)$ & & \\
\hline $\mathrm{T}, \mathrm{n}(\%)$ & & - & - \\
\hline 1 & $92(31.5)$ & & \\
\hline 2 & $66(22.6)$ & & \\
\hline 3 & $57(19.5)$ & & \\
\hline 4 & 77 (26.4) & & \\
\hline $\mathrm{N}, \mathrm{n}(\%)$ & & - & - \\
\hline 0 & $234(80.1)$ & & \\
\hline 1 & $16(5.5)$ & & \\
\hline 2 & $42(14.4)$ & & \\
\hline \multicolumn{4}{|l|}{ M, n (\%) } \\
\hline 0 & 289 (99) & & \\
\hline 1 & $3(1)$ & & \\
\hline $\mathrm{G}, \mathrm{n}(\%)$ & & - & - \\
\hline 1 & $72(25)$ & & \\
\hline 2 & $192(65.8)$ & & \\
\hline 3 & $27(9.7)$ & & \\
\hline
\end{tabular}

*Pearson Chi-Square, **Student's $t$-test. SD: Standard deviation; T: tumor size; M: metastasis; N: metastasis to neck lymph nodes; G: tumor differentiation grade.

\section{Materials and Methods}

Ethics statement. All study procedures were carried out according to the Declaration of Helsinki, and the study protocol was approved by the Kaunas Regional Ethics Committee for Biomedical Research (authorization number: BE-2-37). Objectives and methods of the study were explained to all study subjects prior to the examination. Written informed consent was obtained from all subjects. The study was conducted in the Department of Otorhinolaryngology, Lithuanian University of Health Sciences (LUHS), Kaunas, Lithuania.

Study protocol/design. This case-control study consisted of 300 first time histologically verified LSCC patients [stage I to IV as defined by the American Joint Committee on Cancer (29)] and 369 healthy subjects as a reference (control) group. The control group consisted of healthy volunteers who agreed to take part in this study. Pregnant or breastfeeding women, patients younger than 18 years, patients with systemic comorbidities [e.g., diabetes mellitus, malignant tumors (except LSCC in the case group)] were excluded from the study. Peripheral venous blood samples were collected from all case group patients before treatment at the Department of Otorhinolaryngology, LUHS. Blood samples of the control group subjects were collected in the Outpatient Clinic of LUHS. The 


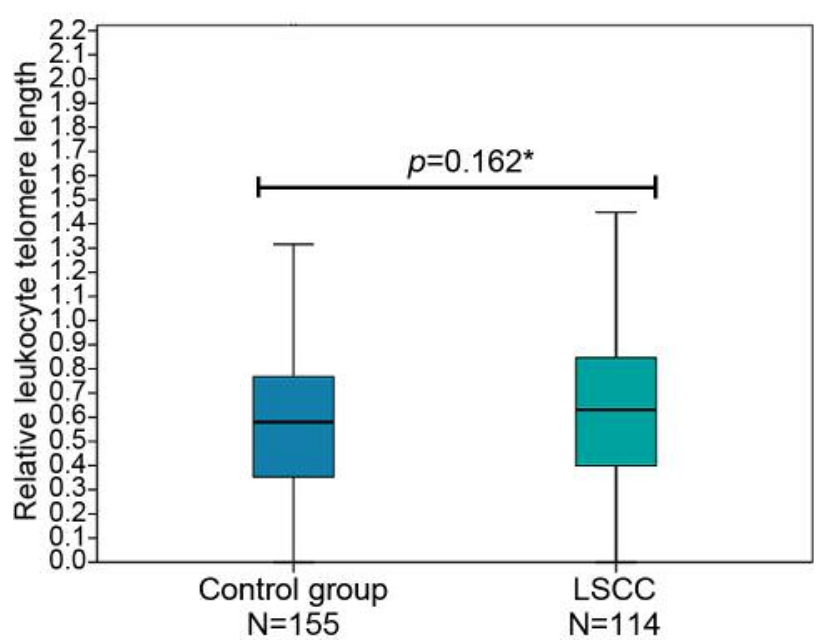

Figure 1. Relative leukocyte telomere length in the LSCC patient and control groups. *Mann-Whitney U-test.

control group was formed adjusting to the demographic characteristics of the LSCC patient group (Table I).

DNA extraction and genotyping. DNA was extracted from venous peripheral blood samples using the DNA salting-out method. The genotyping of all six SNPs was performed using TaqMan ${ }^{\circledR}$ Genotyping assays (Applied Biosystems Foster City, CA, USA): C_26414916_20 (rs2736098), C_1150767_20 (rs401681), C_1869846_10 (rs1545827), C_1869856_10 (rs10107605), C_30418896_20 (rs 10509637), C_29498647_20 (rs 10509639) according to manufacturer's instructions using the real-time polymerase chain reaction (PCR) method.

Relative leukocyte telomere length measurement. Relative LTL for study subjects was determined using modified quantitative real-time PCR (qPCR) protocol (30). The relative LTL for each sample was determined using two separate qPCR runs for each sample, the first to determine the cycle threshold $(\mathrm{Ct})$ value for telomere amplification, and the second one to determine the $\mathrm{Ct}$ value for control gene amplification. A standard curve was generated in each run, consisting of a 6-point serial dilution of the reference DNA pool. The Ct data generated in both runs were used to calculate relative LTL values for each sample according to the formula: $\mathrm{T} / \mathrm{S}=2^{-\Delta \Delta \mathrm{Ct}}$ (31).

Statistical analysis. Statistical analysis was performed using the SPSS/W 20.0 software (Statistical Package for the Social Sciences for Windows, Inc., Chicago, IL, USA). The data of relative LTL are presented as median with interquartile range (IQR) and mean rank. The data of age are presented as mean with standard deviation (SD). Normality of the data distribution was checked using the Shapiro-Wilk test. The Mann-Whitney test was used for comparison of relative LTL between two groups. Student's $t$-test was performed to compare the average age between the study groups. Frequencies of genotypes and alleles, gender, age, distribution of LSCC stage, tumor size, neck lymph nodes, metastasis and tumor differentiation grade are reported using

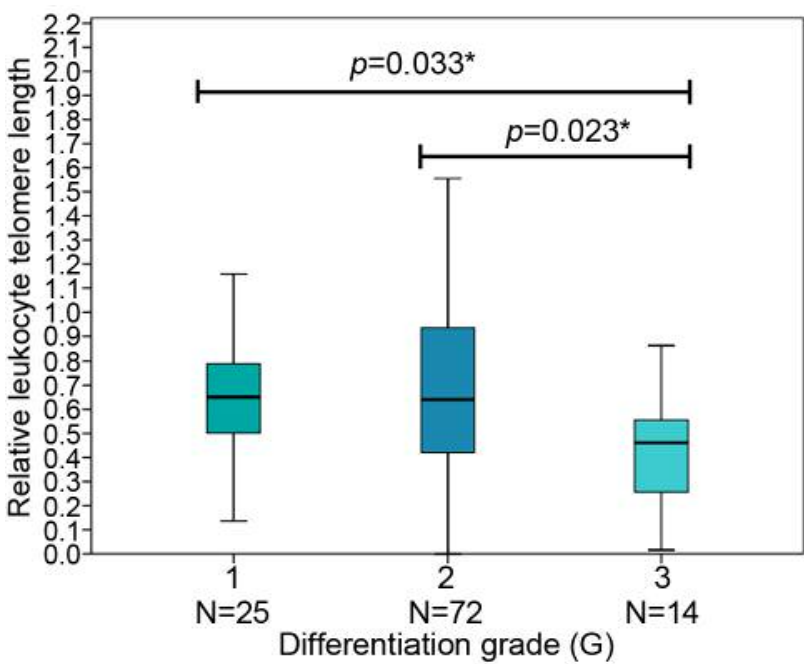

Figure 2. Relative leukocyte telomere length in LSCC patients by tumor differentiation grade $(G)$. *Mann-Whitney U-test.

absolute numbers with percentages. Hardy-Weinberg equilibrium analysis was performed to compare the observed and expected frequencies of TERT (rs2736098, rs401681), TRF1 (rs1545827, rs10107605) and TNKS2 (rs10509637, rs10509639). The distributions of the genotypes and alleles of TERT (rs2736098, rs401681), TRF1 (rs1545827, rs10107605), TNKS2 (rs10509637, rs10509639) as well as gender distribution and long and short telomeres in the LSCC and control groups were compared using the $\chi^{2}$ test. Binomial logistic regression analysis was performed to estimate the impact of genotypes on LSCC development. Odds ratios and 95\% confidence intervals are presented. The selection of the best genetic model was based on the Akaike Information Criterion (AIC); therefore, the best genetic models were those with the lowest AIC values. Differences were considered statistically significant when $p<0.05$.

\section{Results}

The total study group consisted of 300 patients with LSCC: $289(96.3 \%)$ males and $11(3.7 \%)$ females with a median age of 62.8 ( $\mathrm{SD}=8.8)$ years and of 369 healthy subjects: 357 $(96.7 \%)$ males and $12(3.3 \%)$ females with a mean age of 62.5 $(\mathrm{SD}=14.3)$ years. The LSCC patient and control groups were adjusted by age and gender. Demographic characteristics of the total study group are presented in Table I.

Relative LTL was analyzed in 155 healthy subjects: 143 $(92.3 \%)$ males and $12(7.7 \%)$ females, and in 114 patients with LSCC: $110(96.5 \%)$ males and $4(3.5 \%)$ females. No significant differences in relative LTL between the LSCC and control groups were revealed [relative LTL, median $(\mathrm{IQR})=0.5794(0.421)$ vs. $0.6306(0.453), p=0.162]$ (Figure 1).

Comparative analysis of relative LTL in patient subgroups according to the LSCC differentiation grade $(\mathrm{G})$ detected 
Table II. The relative leukocyte telomere length distribution as short and long telomeres in the patients with LSCC and control group subjects.

\begin{tabular}{lccc}
\hline Characteristic & $\begin{array}{c}\text { LSCC group } \\
\mathrm{N}=114\end{array}$ & $\begin{array}{c}\text { Control group } \\
\mathrm{N}=155\end{array}$ & $p$-Value \\
\hline Short telomeres & $50(43.9)$ & $77(49.7)$ & $0.345^{*}$ \\
Long telomeres & $64(56.1)$ & $78(50.3)$ & \\
\hline
\end{tabular}

*Pearson Chi-Square.

Table III. Analysis of Hardy-Weinberg equilibrium in the control group.

\begin{tabular}{lclcr}
\hline SNP & \multicolumn{2}{c}{$\begin{array}{c}\text { Allele } \\
\text { frequencies }\end{array}$} & $\begin{array}{c}\text { Genotype } \\
\text { distribution }\end{array}$ & $p$-Value \\
\hline$T E R T$ rs2736098 & $0.79 \mathrm{C}$ & $0.21 \mathrm{~T}$ & $20 / 117 / 232$ & 0.305 \\
$T E R T$ rs401681 & $0.55 \mathrm{C}$ & $0.45 \mathrm{~T}$ & $63 / 204 / 102$ & $\mathbf{0 . 0 2 3}$ \\
$T R F 1$ rs1545827 & $0.6 \mathrm{C}$ & $0.4 \mathrm{~T}$ & $64 / 169 / 136$ & 0.359 \\
$T R F 1$ rs10107605 & $0.91 \mathrm{~A}$ & $0.09 \mathrm{C}$ & $4 / 61 / 304$ & 0.634 \\
$T N K S 2$ rs10509637 & $0.84 \mathrm{~A}$ & $0.16 \mathrm{G}$ & $8 / 102 / 259$ & 0.578 \\
$T N K S 2$ rs10509639 & $0.87 \mathrm{~A}$ & $0.13 \mathrm{G}$ & $22 / 52 / 295$ & $<\mathbf{0 . 0 0 1}$ \\
\hline
\end{tabular}

Bold value indicates statistical significance.

statistically significantly shorter relative LTL in patients of LSCC G3 subgroup compared to G1 [relative LTL, median $(\mathrm{IQR})=0.4610(0.4331) v s .0 .6508(0.668), p=0.033]$ and $\mathrm{G} 2$ subgroups [relative LTL, median (IQR) $=0.4610(0.4331) v s$. 0.6408 (0.6967), respectively, $p=0.023$ ] (Figure 2).

The relative LTL was divided into two groups - short and long telomeres, based on the median telomere length of control group subjects. The relative LTL lower than the median telomere length of the control group was considered to represent "short telomeres" and the higher "long telomeres". There were no statistically significant differences in distribution of short and long telomeres between the LSCC and control groups (43.9\% and $56.1 \%$ vs. $49.7 \%$ and $50.3 \%, p=0.345$ ] (Table II).

Distributions of the analyzed TERT rs2736098, TERTCLPTM1 rs401681, TRF1 rs1545827, rs10107605 and TNKS2 rs10509637 matched the Hardy-Weinberg equilibrium (HWE) ( $p>0.001$ ). Despite the TNKS2 rs10509639 not matching HWE, we did not exclude this polymorphism from further statistical analysis because such distribution of genotypes and alleles is possible due to the relatively small sample size (Table III).

Distributions of frequencies of the following genotypes and alleles were analyzed in the control and LSCC groups: TERT rs2736098, TERT-CLPTM1 rs401681, TRF1 rs1545827, rs10107605, and TNKS2 rs10509637, rs10509639. Significant differences were found in the genotype distributions of TERT rs 401681 and TNKS2 rs10509639 between the study groups ( $p=0.033$ and $p<0.001$, respectively) (Table IV).
Table IV. Frequencies of genotypes and alleles of TERT rs2736098, rs401681, TRF1 rs1545827, rs10107605, TNKS2 rs10509637 and rs10509639 in the control and patients with LSCC groups.

\begin{tabular}{|c|c|c|c|}
\hline Polymorphism & Control group $\mathrm{n} / \%$ & $\mathrm{LSCC} \mathrm{n} / \%$ & $p$-Value \\
\hline \multicolumn{4}{|l|}{ TERT rs 273609} \\
\hline $\mathrm{C} / \mathrm{C}$ & $232(62.9)$ & $171(57.0)$ & \multirow[t]{4}{*}{0.304} \\
\hline $\mathrm{C} / \mathrm{T}$ & $117(31.7)$ & $110(36.7)$ & \\
\hline $\mathrm{T} / \mathrm{T}$ & $20(5.4)$ & $19(6.3)$ & \\
\hline Total & $369(100)$ & $300(100)$ & \\
\hline \multicolumn{4}{|l|}{ Allele } \\
\hline $\mathrm{C}$ & $581(78.7)$ & $452(75.3)$ & \multirow[t]{2}{*}{0.141} \\
\hline $\mathrm{T}$ & $157(21.3)$ & $148(24.7)$ & \\
\hline \multicolumn{4}{|l|}{ TERT $\mathrm{rs} 401681$} \\
\hline $\mathrm{C} / \mathrm{C}$ & $102(27.6)$ & $108(36.0)$ & \multirow[t]{4}{*}{0.033} \\
\hline $\mathrm{C} / \mathrm{T}$ & $204(55.3)$ & $137(45.7)$ & \\
\hline $\mathrm{T} / \mathrm{T}$ & $63(17.1)$ & $55(18.3)$ & \\
\hline Total & $369(100)$ & $300(100)$ & \\
\hline \multicolumn{4}{|l|}{ Allele } \\
\hline $\mathrm{C}$ & $408(55.3)$ & $353(58.8)$ & \multirow[t]{2}{*}{0.872} \\
\hline $\mathrm{T}$ & $330(44.7)$ & $247(41.2)$ & \\
\hline \multicolumn{4}{|c|}{ TRF1 rs1545827 } \\
\hline $\mathrm{C} / \mathrm{C}$ & $136(36.9)$ & $97(32.3)$ & \multirow[t]{4}{*}{0.243} \\
\hline $\mathrm{C} / \mathrm{T}$ & $169(45.8)$ & $157(52.3)$ & \\
\hline $\mathrm{T} / \mathrm{T}$ & $64(17.3)$ & $46(15.3)$ & \\
\hline Total & $369(100)$ & $300(100)$ & \\
\hline \multicolumn{4}{|l|}{ Allele } \\
\hline $\mathrm{C}$ & $441(59.8)$ & $351(58.5)$ & \multirow[t]{2}{*}{0.642} \\
\hline $\mathrm{T}$ & $297(40.2)$ & $249(41.5)$ & \\
\hline \multicolumn{4}{|c|}{ TRF1 rs10107605 } \\
\hline $\mathrm{A} / \mathrm{A}$ & 304 (82.4) & $241(80.3)$ & \multirow[t]{5}{*}{0.613} \\
\hline $\mathrm{A} / \mathrm{C}$ & $61(16.5)$ & $57(19.0)$ & \\
\hline $\mathrm{C} / \mathrm{C}$ & $4(1.1)$ & $2(0.7)$ & \\
\hline Total & $369(100)$ & $300(100)$ & \\
\hline \multicolumn{3}{|l|}{ Allele } & \\
\hline A & $669(90.7)$ & $539(89.8)$ & \multirow[t]{2}{*}{0.616} \\
\hline $\mathrm{C}$ & $69(9.3)$ & $61(10.2)$ & \\
\hline \multicolumn{4}{|c|}{ TNKS2 rs 10509637} \\
\hline $\mathrm{A} / \mathrm{A}$ & $259(70.2)$ & $196(65.3)$ & \multirow[t]{4}{*}{0.405} \\
\hline $\mathrm{A} / \mathrm{G}$ & $102(27.6)$ & $96(32.0)$ & \\
\hline $\mathrm{G} / \mathrm{G}$ & $8(2.2)$ & $8(2.7)$ & \\
\hline Total & $369(100)$ & $300(100)$ & \\
\hline \multicolumn{4}{|l|}{ Allele } \\
\hline A & $620(84)$ & $488(81.3)$ & \multirow[t]{2}{*}{0.197} \\
\hline $\mathrm{G}$ & $118(16)$ & $112(18.7)$ & \\
\hline \multicolumn{4}{|c|}{ TNKS2 rs 10509639} \\
\hline $\mathrm{A} / \mathrm{A}$ & 295 (79.9) & $242(80.7)$ & \multirow[t]{5}{*}{$<0.001$} \\
\hline $\mathrm{A} / \mathrm{G}$ & $52(14.1)$ & $58(19.3)$ & \\
\hline $\mathrm{G} / \mathrm{G}$ & $22(6.0)$ & $0(0.0)$ & \\
\hline Total & 369 (100) & $300(100)$ & \\
\hline \multicolumn{3}{|l|}{ Allele } & \\
\hline A & $642(87)$ & $542(90)$ & 0.057 \\
\hline $\mathrm{G}$ & $96(13)$ & $58(10)$ & \\
\hline
\end{tabular}

Bold values indicate statistical significance.

To evaluate the impact of TERT rs2736098, rs401681, TRF1 rs1545827, rs10107605 and TNKS2 rs10509637 and rs10509639 on the LSCC development, a binomial logistic regression was applied. Our results revealed that the TERT 
Table V. Binomial logistic regression analysis of TERT rs2736098, rs401681, TRF1 rs1545827, rs10107605, TNKS2 rs10509637 and rs10509639 in the control and patients with LSCC groups.

\begin{tabular}{|c|c|c|c|c|}
\hline Model & Genotype/allele & OR $(95 \% \mathrm{CI})$ & $p$-Value & AIC \\
\hline & & TERT rs2736098 & & \\
\hline \multirow[t]{2}{*}{ Codominant } & $\mathrm{C} / \mathrm{T} v s . \mathrm{C} / \mathrm{C}$ & $1.276(0.960-1.769)$ & 0.144 & 921.921 \\
\hline & $\mathrm{T} / \mathrm{T} v s . \mathrm{C} / \mathrm{C}$ & $1.289(0.667-2.489)$ & 0.450 & \\
\hline Dominant & $\mathrm{C} / \mathrm{T}+\mathrm{T} / \mathrm{T}$ vs. $\mathrm{C} / \mathrm{C}$ & $1.278(0.936-1.744)$ & 0.123 & 919.921 \\
\hline Recessive & $\mathrm{T} / \mathrm{T}$ vs. $\mathrm{C} / \mathrm{C}+\mathrm{C} / \mathrm{T}$ & $1.180(0.618-2.254)$ & 0.616 & 922.051 \\
\hline Overdominant & $\mathrm{C} / \mathrm{T} v s . \mathrm{C} / \mathrm{C}+\mathrm{T} / \mathrm{T}$ & $1.247(0.904-1.719)$ & 0.178 & 920.489 \\
\hline \multirow[t]{2}{*}{ Additive } & $\mathrm{T}$ & $1.204(0.936-1.549)$ & 0.149 & 920.217 \\
\hline & & $T E R T$ rs 401681 & & \\
\hline \multirow[t]{2}{*}{ Codominant } & $\mathrm{C} / \mathrm{T} v s . \mathrm{C} / \mathrm{C}$ & $0.634(0.448-0.897)$ & 0.010 & 917.466 \\
\hline & $\mathrm{T} / \mathrm{T} v s . \mathrm{C} / \mathrm{C}$ & $0.825(0.525-1.295)$ & 0.402 & \\
\hline Dominant & $\mathrm{C} / \mathrm{T}+\mathrm{T} / \mathrm{T} v s . \mathrm{C} / \mathrm{C}$ & $0.679(0.489-0.943)$ & 0.021 & 916.949 \\
\hline Recessive & $\mathrm{T} / \mathrm{T} v s . \mathrm{C} / \mathrm{C}+\mathrm{C} / \mathrm{T}$ & $1.090(0.732-1.625)$ & 0.671 & 922.121 \\
\hline Overdominant & $\mathrm{C} / \mathrm{T} v s . \mathrm{C} / \mathrm{C}+\mathrm{T} / \mathrm{T}$ & $0.680(0.501-0.923)$ & 0.013 & 16.1689 \\
\hline \multirow[t]{2}{*}{ Additive } & $\mathrm{T}$ & $0.860(0.6880-1.074)$ & 0.184 & 920.530 \\
\hline & & TRF1 rs1545827 & & \\
\hline \multirow[t]{2}{*}{ Codominant } & $\mathrm{C} / \mathrm{T} v s . \mathrm{C} / \mathrm{C}$ & $1.303(0.928-1.828)$ & 0.127 & 921.472 \\
\hline & $\mathrm{T} / \mathrm{T} v s . \mathrm{C} / \mathrm{C}$ & $1.008(0.636-1.596)$ & 0.974 & \\
\hline Dominant & $\mathrm{C} / \mathrm{T}+\mathrm{T} / \mathrm{T}$ vs. $\mathrm{C} / \mathrm{C}$ & $1.222(0.886-1.684)$ & 0.222 & 920.806 \\
\hline Recessive & $\mathrm{T} / \mathrm{T}$ vs. $\mathrm{C} / \mathrm{C}+\mathrm{C} / \mathrm{T}$ & $0.863(0.571-1.305)$ & 0.486 & 921.813 \\
\hline Overdominant & $\mathrm{C} / \mathrm{T}$ vs. $\mathrm{C} / \mathrm{C}+\mathrm{T} / \mathrm{T}$ & $1.299(0.957-1.763)$ & 0.093 & 919.473 \\
\hline \multirow[t]{2}{*}{ Additive } & $\mathrm{T}$ & $1.054(0.846-1.313)$ & 0.641 & 922.084 \\
\hline & & TRF1 rs 10107605 & & \\
\hline \multirow[t]{2}{*}{ Codominant } & $\mathrm{A} / \mathrm{C} v s . \mathrm{A} / \mathrm{A}$ & $1.791(0.791-1.756)$ & 0.419 & \\
\hline & $\mathrm{C} / \mathrm{C} v s . \mathrm{A} / \mathrm{A}$ & $0.631(0.115-3.472)$ & 0.596 & 923.317 \\
\hline Dominant & $\mathrm{A} / \mathrm{C}+\mathrm{C} / \mathrm{C} v s . \mathrm{A} / \mathrm{A}$ & $1.145(0.775-1.693)$ & 0.497 & 921.842 \\
\hline Recessive & $\mathrm{C} / \mathrm{C} v s . \mathrm{A} / \mathrm{A}+\mathrm{A} / \mathrm{C}$ & $0.612(0.111-3.367)$ & 0.573 & 921.969 \\
\hline Overdominant & $\mathrm{A} / \mathrm{C} v s . \mathrm{A} / \mathrm{A}+\mathrm{C} / \mathrm{C}$ & $1.184(0.795-1.764)$ & 0.405 & 921.610 \\
\hline \multirow[t]{2}{*}{ Additive } & $\mathrm{C}$ & $1.098(0.763-1.1579)$ & 0.615 & 922.049 \\
\hline & & TNKS2 rs10509637 & & \\
\hline \multirow[t]{2}{*}{ Codominant } & $\mathrm{A} / \mathrm{G} v s . \mathrm{A} / \mathrm{A}$ & $1.244(0.890-1.738)$ & 0.202 & 922.498 \\
\hline & $\mathrm{G} / \mathrm{G} v s . \mathrm{A} / \mathrm{A}$ & $1.321(0.487-3.583)$ & 0.584 & \\
\hline Dominant & $\mathrm{A} / \mathrm{G}+\mathrm{G} / \mathrm{G} v s . \mathrm{A} / \mathrm{A}$ & $1.249(0.902-1.731)$ & 0.181 & 920.512 \\
\hline Recessive & $\mathrm{G} / \mathrm{G} v s . \mathrm{A} / \mathrm{A}+\mathrm{A} / \mathrm{G}$ & $1.236(0.458-3.334)$ & 0.675 & 920.126 \\
\hline Overdominant & $\mathrm{A} / \mathrm{G} v s . \mathrm{A} / \mathrm{A}+\mathrm{G} / \mathrm{G}$ & $1.232(0.883-1.719)$ & 0.220 & 920.797 \\
\hline \multirow[t]{2}{*}{ Additive } & $\mathrm{G}$ & $1.216(0.909-1.626)$ & 0.188 & 920.571 \\
\hline & & $T N K S 2$ rs 10509639 & & \\
\hline \multirow[t]{2}{*}{ Codominant } & $\mathrm{A} / \mathrm{G} v s . \mathrm{A} / \mathrm{A}$ & $1.360(0.901-2.051)$ & 0.143 & 895.366 \\
\hline & $\mathrm{G} / \mathrm{G} v s . \mathrm{A} / \mathrm{A}$ & $0.000(0.000-0.000)$ & 0.998 & \\
\hline Dominant & $\mathrm{A} / \mathrm{G}+\mathrm{G} / \mathrm{G} v s . \mathrm{A} / \mathrm{A}$ & $0.955(0.651-1.402)$ & 0.816 & 922.247 \\
\hline Recessive & $\mathrm{G} / \mathrm{G} v s . \mathrm{A} / \mathrm{A}+\mathrm{A} / \mathrm{G}$ & $0.000(0.000-0.000)$ & 0.998 & 897.515 \\
\hline Overdominant & $\mathrm{A} / \mathrm{G} v s . \mathrm{A} / \mathrm{A}+\mathrm{G} / \mathrm{G}$ & $1.461(0.970-2.202)$ & 0.070 & 921.010 \\
\hline Additive & $\mathrm{G}$ & $0.754(0.549-1.037)$ & 0.083 & 921.215 \\
\hline
\end{tabular}

OR: Odds ratio; CI: confidence interval; $p$-Value: significance level (alpha=0.05); AIC: Akaike information criterion. 
Table VI. Frequencies of genotypes and alleles of TERT rs2736098, $r s 401681$, TRF1 rs 1545827, rs10107605, TNKS2 rs10509637, rs10509639 in the short and long telomeres subgroups.

\begin{tabular}{|c|c|c|c|}
\hline Polymorphisms & $\begin{array}{c}\text { Short telomeres } \\
\mathrm{n} / \%\end{array}$ & $\begin{array}{c}\text { Long telomeres } \\
\mathrm{n} / \%\end{array}$ & $p$-Value \\
\hline \multicolumn{4}{|l|}{ TERT rs 273609} \\
\hline $\mathrm{C} / \mathrm{C}$ & $82(64.6)$ & $81(57.4)$ & \multirow[t]{4}{*}{0.416} \\
\hline $\mathrm{C} / \mathrm{T}$ & $38(29.9)$ & $53(37.6)$ & \\
\hline $\mathrm{T} / \mathrm{T}$ & $7(5.5)$ & $7(5.0)$ & \\
\hline Total & $127(100)$ & $141(100)$ & \\
\hline \multicolumn{4}{|l|}{ Allele } \\
\hline $\mathrm{C}$ & $202(79.5)$ & $215(76.2)$ & \multirow[t]{2}{*}{0.361} \\
\hline $\mathrm{T}$ & $52(20.5)$ & $67(23.8)$ & \\
\hline \multicolumn{4}{|l|}{ TERT rs401681 } \\
\hline $\mathrm{C} / \mathrm{C}$ & $42(33.1)$ & $36(25.5)$ & \multirow[t]{5}{*}{0.325} \\
\hline $\mathrm{C} / \mathrm{T}$ & $63(49.6)$ & $82(58.2)$ & \\
\hline $\mathrm{T} / \mathrm{T}$ & $22(17.3)$ & $23(16.3)$ & \\
\hline Total & $127(100)$ & $141(100)$ & \\
\hline \multicolumn{3}{|l|}{ Allele } & \\
\hline $\mathrm{C}$ & 147 (57.9) & $154(54.6)$ & \multirow[t]{2}{*}{0.447} \\
\hline $\mathrm{T}$ & $107(42.1)$ & $128(45.4)$ & \\
\hline \multicolumn{4}{|l|}{ TRF1 rs1545827 } \\
\hline $\mathrm{C} / \mathrm{C}$ & $45(35.4)$ & $60(42.6)$ & \multirow[t]{5}{*}{0.313} \\
\hline $\mathrm{C} / \mathrm{T}$ & $64(50.4)$ & $58(41.1)$ & \\
\hline $\mathrm{T} / \mathrm{T}$ & $18(14.2)$ & $23(16.3)$ & \\
\hline Total & $127(100)$ & $141(100)$ & \\
\hline \multicolumn{3}{|l|}{ Allele } & \\
\hline $\mathrm{C}$ & $154(60.6)$ & $178(63.1)$ & \multirow[t]{2}{*}{0.553} \\
\hline $\mathrm{T}$ & $100(39.4)$ & $104(36.9)$ & \\
\hline \multicolumn{4}{|c|}{ TRF1 rs10107605 } \\
\hline $\mathrm{A} / \mathrm{A}$ & $107(84.3)$ & $116(82.3)$ & \multirow[t]{5}{*}{0.483} \\
\hline $\mathrm{A} / \mathrm{C}$ & $19(15.0)$ & 25 (17.7) & \\
\hline $\mathrm{C} / \mathrm{C}$ & $1(0.8)$ & $0(0.0)$ & \\
\hline Total & $127(100)$ & $141(100)$ & \\
\hline \multicolumn{3}{|l|}{ Allele } & \\
\hline A & $233(91.7)$ & 257 (91.1) & \multirow[t]{2}{*}{0.805} \\
\hline $\mathrm{C}$ & $21(8.3)$ & $25(8.9)$ & \\
\hline \multicolumn{4}{|c|}{ TNKS2 rs 10509637} \\
\hline $\mathrm{A} / \mathrm{A}$ & $81(63.8)$ & $102(72.3)$ & \multirow[t]{5}{*}{0.182} \\
\hline $\mathrm{A} / \mathrm{G}$ & $39(30.7)$ & $36(25.5)$ & \\
\hline $\mathrm{G} / \mathrm{G}$ & $7(5.5)$ & $3(2.1)$ & \\
\hline Total & $127(100)$ & $141(100)$ & \\
\hline \multicolumn{3}{|l|}{ Allele } & \\
\hline A & $201(79.1)$ & $240(85.1)$ & \multirow[t]{2}{*}{0.071} \\
\hline $\mathrm{G}$ & $53(20.9)$ & $42(14.9)$ & \\
\hline \multicolumn{4}{|c|}{ TNKS2 rs 10509639} \\
\hline $\mathrm{A} / \mathrm{A}$ & $110(86.6)$ & $123(87.2)$ & \multirow[t]{5}{*}{0.986} \\
\hline $\mathrm{A} / \mathrm{G}$ & $14(1)$ & 15 (10.6) & \\
\hline $\mathrm{G} / \mathrm{G}$ & $3(2.4)$ & $3(2.1)$ & \\
\hline Total & $127(100)$ & $64(100)$ & \\
\hline \multicolumn{3}{|l|}{ Allele } & \\
\hline A & $234(92.1)$ & $261(92.5)$ & 0.853 \\
\hline $\mathrm{G}$ & $20(7.8)$ & $21(7.5)$ & \\
\hline
\end{tabular}

rs401681 C/T genotype was associated with approximately $30 \%$ decreased odds of LSCC development in the codominant $(\mathrm{OR}=0.634 ; 95 \% \mathrm{CI}=0.448-0.897 ; p=0.010)$, and overdominant $(\mathrm{OR}=0.680 ; 95 \% \mathrm{CI}=0.501-0.923 ; p=0.013)$ models; $\mathrm{C} / \mathrm{T}+\mathrm{T} / \mathrm{T}$ genotypes were also associated with approximately $30 \%$ decreased odds of LSCC development in the dominant model $(\mathrm{OR}=0.679 ; 95 \% \mathrm{CI}=0.489-0.943$; $p=0.021)$ model (Table V).

Analysis of frequency distribution of genotypes and alleles of TERT rs2736098, rs401681, TRF1 rs1545827, rs10107605 and TNKS2 rs10509637, rs10509639 in the short and long telomeres subgroups revealed no statistically significant differences (Table VI).

\section{Discussion}

Results of several studies have shown that telomere aberrations, mainly consisting of shortening, are consistently found in head and neck squamous cell carcinoma (HNSCC) precursors and in the mucosa surrounding pre-neoplastic areas, both in invasive carcinomas and in peripheral venous blood, supporting the hypothesis that genomic instability driven by telomere dysfunction is an early event in the HNSCC oncogenic process (32-36).

Data in literature regarding the associations of relative LTL and development of HNSCC are rather scarce and controversial. Several studies have observed that patients with short telomeres have a higher risk of developing HNSCC (34-37). Namely, short relative LTL was associated with an increased risk of developing oral premalignant lesions and oral squamous cell carcinoma, suggesting that patients with premalignant lesions and short relative LTL face an increased risk to develop this type of malignant tumor (38).

Only a few reports have measured relative LTL in blood cells collected from HNSCC patients. Liu et al. have not found a significant association between patients' relative LTL in peripheral blood cells and the risk of developing HNSCC in one large case-control study (39). On the other hand, other studies have revealed shortened telomeres in peripheral blood leukocytes obtained from patients with HNSCC compared to healthy controls. As found by Alves-Paiva et al. in a Brazilian cohort of HNSCC patients, the median relative LTL was lower in HNSCC patients compared to age-matched healthy individuals, and this was associated with increased risk of HNSCC (14). Similarly, Barczak et al. have reported the presence of shorter telomeres even in early stage HNSCC tumors (40). Xun Zhu et al. performed a meta-analysis in 2016 , to assess the total cancer risk or cancer-specific risk associated with telomere length. A total of 23,379 cancer cases and 68,792 controls from 51 independent publications were included in this study. They did not find an association between telomere length and overall cancer risk but demonstrated a significant relationship with short telomeres and increased risk of HNSCC (41). The discrepancy between literature data may be explained by significant diversity of malignances united under the umbrella of the HNSCC term, 
as this includes malignant tumors of different localizations (oral, pharyngeal, laryngeal regions) as well as of different biological and clinical behavior. All these different tumors have different etiology and pooling all cancer types together may mask the significant associations of relative LTL with individual cancer types (38). In the present study, a pure cohort of LSCC patients was investigated. Results revealed no statistically significant differences in relative LTL between the LSCC patients and control groups. Presumably, these findings reflect the biological and clinical peculiarities of the LSCC tumor.

No data on the relation between relative LTL and tumor differentiation grade in LSCC have been published so far. In the present study, shorter relative LTL in the LSCC G3 subgroup against the G2 and G1 subgroups were detected for the first time. It is generally accepted that G3 tumor differentiation grade is usually associated with the worst prognosis (early metastasis and worst overall survival) when compared to G1 and G2 (42). On the other hand, there is an evidence from various studies that shortened telomeres are related to increased mortality rate in general (43). Therefore, data of the present study on telomere shortening in the subgroup of G3 LSCC patients are in concordance with this concept.

Telomere length regulating factors such as the telomerase complex, the shelterin complex and tankyrase as well as coding genes' polymorphisms are considered important factors in cancerogenesis (15). In the present study, frequencies of genotypes and alleles of TERT rs2736098, rs401681, TRF1 rs1545827, rs10107605, TNKS2 rs 10509637 and rs 10509639 were analyzed in the LSCC patients and control groups. Significant differences were found in genotype distributions of TERT rs401681 and TNKS2 rs 10509639 between the study groups $(p=0.033$ and $p<0.001$, respectively). Moreover, after performing binomial logistic regression, we found that the TERT rs401681 C/T and $\mathrm{C} / \mathrm{T}+\mathrm{T} / \mathrm{T}$ genotypes were associated with approximately $30 \%$ decreased odds of LSCC development.

According to the literature, SNPs of TERT and TERT $C L P T M 1 L$ at the $5 \mathrm{p} 15.33$ locus have a significant relationship with cancer risk (17-21). A recent study of the Southeast Iranian population has found a protective effect of an allele of TERT rs2736098 against breast cancer (44). Yu et al. have reported results similar to our study. They performed a casecontrol study including 490 cases (histologically diagnosed primary squamous cell carcinomas of the pharynx-larynx) and 590 controls. Study results revealed that the CT and $\mathrm{CT}+\mathrm{CC}$ genotype models of rs 401681 were related to a reduced risk of pharynx-larynx cancer (22). The earlier study by Liu et al. has found that rs401681 CT+TT genotypes lowered the risk of oropharyngeal cancer for smokers and alcohol users (45). Previous studies have investigated the interactions of other SNPs of the TERT gene with head and neck cancer, but no associations have been found $(39,46)$.
Qu et al. have compared the SNPs of TERT C228T and $C 250 T$ between a laryngeal cancer and control group and haven't found any difference; however TERT C250T mutation indicated the worst overall survival rate (46). Liu et al. have investigated a large group of 888 subjects diagnosed with HNSCC but have not found any associations between SNPs of TERT (rs2735940; rs2736098; rs2736109; rs2853669; rs2853677 and rs2853690) and development of this tumor (39). However, results of the present study demonstrated that TERT rs401681 CT and $\mathrm{C} / \mathrm{C}+\mathrm{T} / \mathrm{T}$ genotypes were associated with approximately $30 \%$ decreased odds of LSCC development.

The strength of the present study consists of a relatively large and homogenous group of pure LSCC patients and ageand gender-matched controls. This peculiarity allowed us to perform a precise analysis of associations between relative LTL and LSCC development, with the latter being a specific tumor in one anatomical region. As generally accepted from clinical practice, LSCC (particularly, the glottis cancer) features a relatively low aggressiveness, i.e. low metastatic and spreading rate compared to other malignancies of head and neck region $(47,48)$. Therefore, the absence of differences in relative LTL between the low aggressive tumor patient and age-matched control groups is comprehensible. Moreover, an increased aggressiveness of LSCC, as in the G3 LSCC patient subgroup, reflected significantly decreased relative LTL compared to the G1 and G2 subgroups. Of note is the fact that results of the present study revealed that gene polymorphism TERT rs401681 and its $\mathrm{C} / \mathrm{T}$ and $\mathrm{C} / \mathrm{T}+\mathrm{T} / \mathrm{T}$ genotypes are associated with approximately $30 \%$ reduced odds of LSCC developing. To the best of our knowledge, this is the first report that associates the relative LTL and SNPs of telomere length-associated genes in a pure and homogenous LSCC patient cohort. However, limitations of the present study should be also considered as environmental factors, smoking and alcohol consumption were not analyzed. However, this is foreseen as a task for future research.

\section{Conclusion}

Results of the present study revealed no statistically significant differences in relative LTL between the LSCC patient and control groups. However, the relative LTL was shorter in the G3 subgroup compared to the G2 and G1 subgroups of LSCC patients showing their possible role in tumor development. Also, we found that the genetic marker TERT rs 401681 and its $\mathrm{C} / \mathrm{T}$ and $\mathrm{T} / \mathrm{T}$ genotypes are associated with decreased odds of overall LSCC development.

\section{Conflicts of Interest}

The Authors declare no potential conflicts of interest associated with this study. 


\section{Authors' Contributions}

R.L., A.V., G.G. and V.U. designed research; V.L. and V.U. collected patients' samples; P.V., G.G. and A.V. performed research; R.L., A.V., G.G. and P.V. analyzed data; and P.V., R.L., V.L. and V.U. wrote the paper.

\section{References}

1 Ferlay J, Steliarova-Foucher E, Lortet-Tieulent J, Rosso S, Coebergh JWW, Comber H, Forman D and Bray F: Reprint of: Cancer incidence and mortality patterns in Europe: Estimates for 40 countries in 2012. Eur J Cancer 51(9): 1201-1202, 2015. PMID: 23485231. DOI: 10.1016/j.ejca.2015.05.004

2 Lin HW and Bhattacharyya N: Staging and survival analysis for nonsquamous cell carcinomas of the larynx. Laryngoscope 118(6): 1003-1013, 2008. PMID: 18388773. DOI: 10.1097/ MLG.0b013e3181671b3d

3 National Cancer Institute: Cancer Stat Facts: Laryngeal Cancer. Available from: https://seer.cancer.gov/statfacts/html/laryn.html

4 Tamura Y, Takubo K, Aida J, Araki A and Ito H: Telomere attrition and diabetes mellitus. Geriatr Gerontol Int 16(1): 66-74, 2016. PMID: 27018285. DOI: 10.1111/ggi.12738

5 Oh B, Kim H, Park YN, Yoo JE, Choi J, Kim K, Lee JJ and Park $\mathrm{C}$ : High telomerase activity and long telomeres in advanced hepatocellular carcinomas with poor prognosis. Lab Invest 88(2): 144-152, 2008. PMID: 18158557. DOI: $10.1038 /$ labinvest.3700710

6 Gu Y, Yu C, Miao L, Wang L, Xu C, Xue W, Du J, Yuan H, Dai $\mathrm{J}$, Jin $\mathrm{G}, \mathrm{Hu} \mathrm{Z}$ and $\mathrm{Ma} \mathrm{H}$ : Telomere length, genetic variants and risk of squamous cell carcinoma of the head and neck in Southeast Chinese. Nat Publ Gr 6(20675): 1-7, 2016. PMID: 26857734. DOI: $10.1038 /$ srep20675

7 Gedvilaite G, Vilkeviciute A and Kriauciuniene L: The relationship between leukocyte telomere length and TERT, TRF1 single nucleotide polymorphisms in healthy people of different age groups. Biogerontology 21(1): 57-67, 2020. PMID: 31646401. DOI: $10.1007 / \mathrm{s} 10522-019-09843-0$

8 Danese E and Lippi G: Telomere length: is the future in our " ends "? Ann Transl Med 6(13): 6-8, 2018. PMID: 30094266. DOI: $10.21037 / \mathrm{atm} .2018 .06 .24$

9 de Lange T: Shelterin: the protein complex that shapes and safeguards human telomeres. Genes Dev 19(18): 2100-2110, 2005. PMID: 16166375. DOI: $10.1101 / \mathrm{gad} .1346005$

10 Smith EM, Pendlebury DF and Nandakumar J: Structural biology of telomeres and telomerase. Cell Mol Life Sci 77(1): 61-79, 2019. PMID: 31728577. DOI: 10.1007/s00018-01903369-x

11 Muraki K, Nyhan K, Han L and John P: Mechanisms of telomere loss and their consequences for chromosome instability. Front Oncol 2(135): 1-13, 2012. PMID: 23061048. DOI: 10.3389/fonc. 2012.00135

12 Maciejowski $\mathbf{J}$ and Lange T De: Telomeres in cancer: tumour suppression and genome instability. Nat Publ Gr 18(3): 175-186, 2017. PMID: 28096526. DOI: 10.1038/nrm.2016.171

13 Cleal K, Norris K and Baird D: Telomere length dynamics and the evolution of cancer genome architecture. Int J Mol Sci 19(2): 1-17, 2018. PMID: 29415479. DOI: 10.3390/ijms19020482

14 Alves-paiva RM, Gutierrez-rodrigues F, Pereira-martins DA, Livingstone D, Figueiredo A, Clé D V, Carlos L, Celso R,
Mamede M and Calado RT: Short telomere length in peripheral blood leukocytes in head and neck cancer: Findings in a Brazilian cohort. Head Neck 41(3): 672-677, 2019. PMID: 30589155. DOI: $10.1002 /$ hed 25472

15 Naing C, Aung K, Lai PK and Mak JW: Association between telomere length and the risk of colorectal cancer: A metaanalysis of observational studies. BMC Cancer 17(1): 1-7, 2017. PMID: 28056862. DOI: 10.1186/s12885-016-2997-3

16 Wyatt HDM, West SC and Beattie TL: InTERTpreting telomerase structure and function. Nucleic Acids Res 38(17): 5609-5622, 2010. PMID: 20460453. DOI: 10.1093/nar/gkq370

17 Yuan X, Cheng G, Yu J, Zheng S, Sun C, Sun Q, Li K, Lin Z, Liu T, Li P, Xu Y, Kong F, Bjorkholm M and Xu D: The TERT promoter mutation incidence is modified by germline TERT rs2736098 and rs2736100 polymorphisms in hepatocellular carcinoma. Oncotarget 8(14): 23120-23129, 2017. PMID: 28416747. DOI: $10.18632 /$ oncotarget. 15498

18 Xiao X and He W: Genetic polymorphisms in the TERTCLPTM11 region and lung cancer susceptibility in Chinese males. Oncol Lett 14(2): 1588-1594, 2017. PMID: 28789383. DOI: $10.3892 / \mathrm{ol} .2017 .6289$

19 Ede C, Chen X, Lin M and Chen YY: Sequence variants at the TERT- CLPTM1L locus associate with many cancer types. Nat Genet 41(2): 221-227, 2009. PMID: 19151717. DOI: $10.1038 / \mathrm{ng} .296$

20 Wang Y, Broderick P, Webb E, Wu X, Vijayakrishnan J, Qureshi M, Dong Q, Gu X, Chen WV and Margaret R: Common 5p15.33 and $6 \mathrm{p} 21.33$ variants influence lung cancer risk. Nat Genet 40(12): 1407-1409, 2008. PMID: 18978787. DOI: 10.1038/ng.273

21 Mckay JD, Hung RJ, Gaborieau V, Boffetta P, Byrnes G, Zaridze D, Mukeria A, Szeszenia- N, Lissowska J, Rudnai P, Fabianova E, Mates D, Bencko V, Foretova L, Janout V, Mclaughlin J, Montpetit A, Narod S, Krokan HE, Skorpen F, Elvestad MB, Vatten L, Njølstad I, Axelsson T and Chen C: Lung cancer susceptibility locus at 5p15.33. Nat Genet 40(12): 1404-1406, 2008. PMID: 18978790 . DOI: 10.1038/ng.254.

$22 \mathrm{Yu}$ J, Li X, Zhou B and Yan A: Polymorphisms of the TERTCLPTM1L gene are associated with pharynx-larynx cancer. DNA Cell Biol 38(9): 1-7, 2019. PMID: 31429604. DOI: 10.1089/dna.2019.4744

23 Rice C and Skordalakes E: Structure and function of the telomeric CST complex. Comput Struct Biotechnol J 14(14): 161-167, 2016. PMID: 27239262. DOI: 10.1016/j.csbj.2016.04.002

24 Hosokawa K and Arai F: The role of telomere binding molecules for normal and abnormal hematopoiesis. Int J Hematol 107(6): 646-655, 2018. PMID: 29550946. DOI: 10.1007/s12185-0182432-4

25 Haikarainen T, Krauss S and Lehtio L: Tankyrases: structure, function and therapeutic implications in cancer. Curr Pharm Des 20(41): 6472-6488, 2014. PMID: 24975604. DOI: $10.2174 / 1381612820666140630101525$

26 Jones M, Bisht K, Savage SA, Nandakumar J, Keegan CE and Maillard I: The shelterin complex and hematopoiesis. J Clin Invest 126(5): 1621-1629, 2016. PMID: 27135879. DOI: $10.1172 / \mathrm{JCI} 84547$

27 Varadi V, Brendle A, Brandt A, Johansson R, Enquist K, Henriksson R, Svenson U, Tavelin B, Roos G, Hemminki K, Lenner P and Forsti A: Polymorphisms in telomere-associated genes, breast cancer susceptibility and prognosis. Eur J Cancer 45(17): 30083016, 2009. PMID: 19766477. DOI: 10.1016/j.ejca.2009.08.012 
28 Wang Y, Jiang W, Liu X and Zhang Y: Tankyrase 2 (TNKS2) polymorphism associated with risk in developing non-small cell lung cancer in a Chinese population. Pathol Res Pract 211(10): 766-771, 2015. PMID: 26293798. DOI: 10.1016/j.prp.2015. 07.003

29 American Joint Committee on Cancer: AJCC Cancer Staging Manual, Eighth Edition, 2018. Available from: https:// cancerstaging.org/references-tools/deskreferences/Documents/ AJCC\%20Cancer\%20Staging\%20Form\%20Supplement.pdf

30 Cawthon RM: Telomere measurement by quantitative PCR. Nucleic Acids Res 30(10): 1-6, 2002. PMID: 12000852. DOI: 10.1093/nar/30.10.e47

31 Livak KJ and Schmittgen TD: Analysis of relative gene expression data using real- time quantitative PCR and the $2^{-\Delta \Delta C T}$ method. Methods 25(4): 402-408, 2001. PMID: 11846609. DOI: $10.1006 /$ meth.2001.1262

32 Hou L, Zhang X, Gawron AJ and Liu J: Surrogate tissue telomere length and cancer risk: Shorter or Longer ? Cancer Lett 319(2): 130-135, 2012. PMID: 22269209. DOI: 10.1016/j.canlet.2012.01.028

33 Meeker AK, Hicks JL, Iacobuzio-donahue CA, Montgomery EA, Westra WH, Chan TY, Ronnett BM and Marzo AM De: Telomere length abnormalities occur early in the initiation of epithelial carcinogenesis. Clin Cancer Res 10(10): 3317-3326, 2004. PMID: 15161685. DOI: 10.1158/1078-0432.CCR-0984-03

34 Aida J, Kobayashi T, Saku T, Yamaguchi M, Shimomura N, Nakamura K, Ishikawa N, Maruyama S, Cheng J, Poon SSS, Arai $\mathrm{T}$ and Takubo K: Short telomeres in an oral precancerous lesion: Q-FISH analysis of leukoplakia. J Oral Pathol Med 41(5): 372-378, 2011. PMID: 22122732. DOI: 10.1111/j.16000714.2011.01120.x

35 Aida J, Izumo T, Shimomura N, Nakamura K, Ishikawa N, Matsuura M, Poon SS, Fujiwara M, Sawabe M, Arai T and Takubo K: Telomere lengths in the oral epithelia with and without carcinoma. Eur J Cancer 46(2): 430-438, 2009. PMID: 19910186. DOI: 10.1016/j.ejca.2009.10.018

36 Boscolo-rizzo P, Rampazzo E, Perissinotto E, Assunta M, Giunco S, Baboci L, Spinato G, Spinato R, Tirelli G, Cristina M, Mosto D, Del A and Rossi A De: Telomere shortening in mucosa surrounding the tumor: Biosensor of field cancerization and prognostic marker of mucosal failure in head and neck squamous cell carcinoma. Oral Oncol 51(5): 500-507, 2015 PMID: 25771075. DOI: 10.1016/j.oraloncology.2015.02.100

37 Boscolo-rizzo P, Cristina M, Mosto D, Rampazzo E, Giunco S, Mistro A Del, Menegaldo A, Baboci L, Mantovani M, Tirelli G and Rossi A De: Telomeres and telomerase in head and neck squamous cell carcinoma: from pathogenesis to clinical implications. Cancer Metastasis Rev 35(3): 457-474, 2016. PMID: 27501725. DOI: 10.1007/s10555-016-9633-1

38 Bau D, Lippman SM, Xu E, Gong Y and Lee JJ: Short telomere lengths in peripheral blood leukocytes are associated with an increased risk of oral premalignant lesion and oral squamous cell carcinoma. Cancer 119(24): 4277-4283, 2013. PMID: 24105340. DOI: $10.1002 / \mathrm{cncr} .28367$
39 Liu Z, Ma H, Wei S, Li G, Sturgis EM and Wei Q: Telomere length and TERT functional polymorphisms are not associated with risk of squamous cell carcinoma of the head and neck. Cancer Epidemiol Biomarkers Prev 20(12): 2642-2645, 2011. PMID: 21994403. DOI: 10.1158/1055-9965.EPI-11-0890

40 Barczak W, Suchorska WM, Sobecka A, Bednarowicz K, Machczynski P, Golusinski P, Rubis B, Masternak MM and Golusinski W: hTERT C250T promoter mutation and telomere length as a molecular markers of cancer progression in patients with head and neck cancer. Mol Med Rep 16(1): 441-446, 2017. PMID: 28535013. DOI: 10.3892/mmr.2017.6590

41 Zhu X, Han W, Xue W, Zou Y, Xie C, Du J and Jin G: The association between telomere length and cancer risk in population studies. Sci Rep 6(22243): 1-10, 2016. PMID: 26915412. DOI: $10.1038 /$ srep22243

42 Johansen L V, Grau C and Overgaard J: Laryngeal carcinomamultivariate analysis of prognostic factors in 1252 consecutive patients treated with primary radiotherapy. Acta Oncol 42(7): 771778, 2003. PMID: 14690164. DOI: 10.1080/02841860310017595

43 Wang Q, Zhan Y, Pedersen NL, Fang F and Hägg S: Telomere Length and All-Cause Mortality: A Meta-analysis. Ageing Res Rev 48: 11-20, 2018. PMID: 30254001. DOI: 10.1016/j.arr.2018.09.002

44 Hashemi M, Amininia S, Ebrahimi M, Hashemi SM, Taheri M and Ghavami S: Association between hTERT polymorphisms and the risk of breast cancer in a sample of Southeast Iranian population. BMC Res Notes 7(1): 1-8, 2014. PMID: 25491902. DOI: $10.1186 / 1756-0500-7-895$

45 Liu Z, Li G, Wei S, Niu J, Wang L, Sturgis EM and Wei Q: Genetic variations in TERT - CLPTM1L genes and risk of squamous cell carcinoma of the head and neck. Carcinogenesis 31(11): 1977-1981, 2010. PMID: 20802237. DOI: $10.1093 /$ carcin/bgq179

46 Qu Y, Dang S, Wu K, Shao Y, Yang Q, Ji M, Shi B and Hou P: TERT promoter mutations predict worse survival in laryngeal cancer patients. Int J Cancer 135(4): 1008-1010, 2014. PMID: 24436132. DOI: $10.1002 / \mathrm{ijc} .28728$

47 Cadoni G, Giraldi L, Petrelli L, Pandolfini M, Giuliani M, Paludetti G, Pastorino R, Leoncini E, Arzani D, Almadori G and Boccia S: Prognostic factors in head and neck cancer: a 10-year retrospective analysis in a single-institution in Italy. Acta Otorhinolaryngol Ital 37(6): 458-466, 2017. PMID: 28663597. DOI: $10.14639 / 0392-100 X-1246$

48 Gatta G, Botta L, Sánchez MJ, Anderson LA, Pierannunzio D, Licitra L and EUROCARE Working Group: Prognoses and improvement for head and neck cancers diagnosed in Europe in early 2000s: The EUROCARE-5 population-based study. Eur J Cancer 51(15): 2130-2143, 2015. PMID: 26421817. DOI: 10.1016/j.ejca.2015.07.043
Received April 30, 2020

Revised May 9, 2020

Accepted May 14, 2020 